\title{
Geochemical Bioenergetics and Microbial Metabolisms at Three Contrasting Sites of Serpentinization
}

\author{
COOK, M.C. ${ }^{1}$, MORRILL, P.L. ${ }^{1}$, SUZUKI, S. ${ }^{2}$, BLANK, J.G. ${ }^{3}$
}

${ }^{1}$ Memorial University, St. John's, NL, CAN

$<$ t52mcc@mun.ca>

${ }^{2}$ Kochi Institute for Core Sample Research, JAMSTEC, Nankoku, Kochi 783-8502, Japan

${ }^{3}$ NASA Ames Research Center \& Blue Marble Space

Institute of Science, Mountain View, CA, USA

Serpentinization, the process whereby ultramafic rocks are hydrated, produces serpentine minerals, hydrogen gas, ultra-basic reducing fluids, and methane. These reactions create large amounts of chemical potential that could be exploited by microbial life. Serpentinization may be, or may have been, active in the subsurface of Mars. To better understand the potential habitability of the Martian subsurface biogeochemistry and its capacity to support microbial life, three contrasting Mars analog sites of serpentinization were studied: The Cedars (CA, USA), Aqua de Ney (CA, USA), and Tablelands (NL, CAN).

For each analog site, energy available for microbial utilization was quantified through the calculation of chemical affinities $\left(A_{r}\right)$. The $A_{r}$ values in all of the sites showed that methanogenesis, carbon monoxide oxidation, and methanotrophy should provide the most energy for life and microbial growth. All three of these metabolic processes were tested for in laboratory microcosm experiments. Methane oxidation was not observed in any of the experiments. Microbial methanogenesis was observed in The Cedars microcosms, despite the addition of electron acceptors, but not in any of the other experiments. Carbon monoxide utilization was observed in the Tablelands experiments and not in the other experiments. These results suggest that locations of serpentinization may provide habitable enclaves on other planets while also demonstrating that some of the anticipated life, on Earth, has not adapted to these extreme conditions. 